\title{
DEVELOPMENT OF A DEVICE FOR DETERMINING WORK ELECTRON OUTPUT
}

\author{
Makeeva O.V. ${ }^{1}$, Oleshko V.S. ${ }^{2}$, Fedorov A.V. $^{2}$, Yurov V.M. ${ }^{3}$ \\ 1Moscow State University of Technologies and Management, Moscow, Russia, makeeva-oks@yandex.ru \\ 2 Moscow Aviation Institute (National Research University), Moscow, Russia, ovs_mai@mail.ru \\ ${ }^{3}$ E.A. Buketov Karaganda State University, Karaganda, Kazakhstan, exciton@list.ru
}

\begin{abstract}
The surface is the most important component of metal parts and it is necessary to control its condition at all stages of the life cycle of the part - during its production, operation and repair. To carry out reliable non-destructive testing of metal parts, specially developed tools and methods for its use are necessary. The measuring electrode of the sensor of the measuring device of the contact potential difference and the surface of the metal part to be controlled form a capacitor, between the plates of which the contact potential difference occurs. The sensor of the device is a kind of intermediary between the contact potential difference and the measuring circuit. The portable complex for measuring the contact potential difference developed by the authors on the basis of a portable digital oscilloscope allows non-destructive testing of the surface of metal parts during their production, operation or repair to be performed continuously.
\end{abstract}

Keywords: non-destructive testing, metal part, surface, capacitor, contact potential difference.

\section{Introduction}

In modern engineering, multicomponent alloys based on $\mathrm{Al}, \mathrm{Fe}, \mathrm{Ti}, \mathrm{Cu}, \mathrm{Ni}, \mathrm{Cr}$, and other metals are widely used as materials for machine parts [1]. The surface is the most important component of metal parts and it is necessary to control its condition at all stages of the life cycle of the part - during its production, operation and repair. The surface layer of metal parts can be considered as part of a solid body, which includes its constituent molecules, atoms, protons, neutrons, electrons, vacancies, dislocations, and other components, each of which has energy. Surface conduction electrons are constantly in diffuse motion without leaving the surface in vacuum at a low temperature. However, with increasing temperature, the potential energy can reach such a value that the electron can leave the metal surface. Part of the electrons constantly jumps off the surface of the metal, but then returns back, because its potential energy is insufficient to fly over the potential barrier. It turns out that the metal surface is surrounded by an electronic cloud and electric forces are directed inside the metal surface. To overcome these forces and leave the metal surface, you need to do a job called electron work function (EWF). To determine the EWF you need to measure the potential difference (CPD).

The theory of CPD is directly related to the zone theory of solids. Considering the contact of two metals with different electron work function, we find that the electrons of the upper metal levels with a lower work function (EWF) will be located at low levels of the second metal with a larger work function (REE). As a result of this, the first metal will be charged positively, and the second will be negatively charged. In this case, the energy levels will shift, since the Fermi levels of both metals coincide. Also, the functions of the metals remain constant, and the potential energy of these metals at points outside their surface will differ. These differences lead to the appearance of a potential difference. To assess the state of energy of a metal surface, one needs to measure CPD. When we know the energy state of a metal surface, this is necessary for coating, welding of various metals and more. Knowledge of the energy state of the surface of metal parts is important, for 
example, when applying protective coatings, bonding, soldering, welding and other technological operations.

\section{Materials and methods}

To carry out reliable non-destructive testing of metal parts, specially developed tools and methods for its use are necessary. The measuring electrode (ME) of the sensor of the measuring device of the CPD and the surface of the controlled metal part form a capacitor (DC), between which the CPD occurs. The sensor of the device is, in a way, an intermediary between the CPD and the measuring circuit.

The following functional requirements apply to capacitive sensors:

1. An easy-to-analyze form of dependence between input and output characteristics, preferably linear.

2. Sufficient sensitivity. Our studies have shown that, due to the energy heterogeneity of the surface of metal parts of machines, it is necessary to ensure the sensitivity of the measuring device of the measurement factor of $1 \mathrm{mV}$.

3. Stability of characteristics, the least influence of environmental parameters (humidity, temperature, atmospheric pressure, electromagnetic fields, radiation, etc.). In order to fulfill this requirement, it is necessary to use shielding of the sensor from spurious interference and to use metal having stable characteristics over time as an ME material. Most often, the developers of measuring devices for $\mathrm{CPD}$ use $\mathrm{Au}, \mathrm{Ni}$, or $\mathrm{Cu}$ [2-7].

4. Short measurement time of the CPD.

5. Ease of installation and operation.

6. Maintainability.

7. Low cost.

The indicated properties must be achieved when creating a measuring device for CPD.

The advantages of the method of DC method of the CPD are:

- the capacitor can be manufactured with high accuracy;

- the capacitor has low losses (the passage of electric current through capacitive resistance is not accompanied by heat loss) and a high efficiency;

- small effect of electric forces on the mechanical parts of the capacitor;

- the shape of the capacitor type sensor can be adapted to various surface shapes of the QS.

The disadvantage of capacitor methods for measuring physical quantities are small capacitance values. Therefore, it is necessary to use power amplifiers of the electric signal, or high-frequency oscillations of the capacitor plates, which does not always ensure the stability of the readings of the measuring device. In addition, the measurement error of the CPD can increase the electromagnetic interference arising in the circuit as a result of the remote location from the ME of the signal amplifier [8]. Therefore, it is advisable to place the preliminary signal amplifier directly in the sensor of the measuring device.

\section{Discussion of results}

The quality of the capacitor with alternating electric current (which occurs with DC) is best characterized by the time constant (product of capacitance and parallel resistance) $Q_{\mathrm{V}}[8]$ :

$$
\mathrm{Q}_{\mathrm{V}}=\omega \cdot \mathrm{R} \cdot \mathrm{C}=\omega \cdot \mathrm{T}
$$

where $\omega=2 \pi \mathrm{F}$ is the circular frequency of the alternating current, $\mathrm{rad} / \mathrm{s} ; \mathrm{F}$ is the frequency of alternating electric current, $\mathrm{Hz} ; \mathrm{R}$ - resistance, Ohm; $\mathrm{C}$ is the capacitance of the capacitor, F; T period, s. 
The resistance $\mathrm{R}$ at a high circular frequency $\omega$ is determined not only by insulation, but also includes other losses, for example, dielectric hysteresis losses, and therefore, according to formula (1), it increases in proportion to the circular frequency $\omega$ [8]. The vibrating ME of the sensor that implements the DC principle included in the alternating current circuit has the resistance $\mathrm{R}$ the lower, the higher the frequency $\mathrm{F}$ of the alternating current according to formula (1) [8]. The capacitance of the capacitor, the plates of which form the controlled part and measuring electrode (ME), depends on their shape (we chose a flat ME), the overlap area, dielectric constant (in our case, this is air) and the distance between its plates [8].

With the formation of ME and controlled by the metal part of the capacitor, the plates of which they are, the charge of the capacitor Q can be determined on the basis of the following formula [9]:

$$
\mathrm{Q}=\mathrm{C} \cdot \mathrm{U}=\frac{\varepsilon_{0} \cdot \varepsilon \cdot \mathrm{S} \cdot \mathrm{U}}{\mathrm{d}}
$$

where $\varepsilon_{0} \approx 8.8542 \cdot 10^{-12} \mathrm{~F} / \mathrm{m}$ is the electric constant (dielectric constant of vacuum); $\varepsilon$ is the relative dielectric constant, for dry air $\varepsilon \approx 1,0006$; $\mathrm{S}$ is the area of $\mathrm{ME}, \mathrm{m}^{2}$; $\mathrm{U}$ is the voltage across the capacitor; $d$ is the gap between $\mathrm{ME}$ and capacitor plates $(\mathrm{CO}), \mathrm{m}$

In the case of a DC, the plates of which are ME vibrating with a frequency $\omega$ and the surface of the $\mathrm{CO}$, then the capacitance $\mathrm{S}_{\mathrm{din}}$ will change according to the law [9]:

$$
\mathrm{C}_{\mathrm{din}}(\mathrm{t})=\frac{\varepsilon_{0} \cdot \varepsilon \cdot \mathrm{S}}{\mathrm{d}_{0}+\mathrm{d}_{1} \cdot \sin (\omega \mathrm{t})}=\mathrm{C} \cdot \frac{1}{1+\mathrm{m} \cdot \sin (\omega \mathrm{t})}
$$

where $\mathrm{d}_{0}$ is the average distance between the ME and the surface of the controlled metal part; $\mathrm{d}_{1}$ is the vibration amplitude of the ME; $t$ is the time; $m=d_{1} / d_{0}$ is the modulation coefficient of the DC.

In this case, an electric current I(t) will occur in the circuit [9]:

$$
\mathrm{I}(\mathrm{t})=\mathrm{U} \cdot \frac{\partial \mathrm{C}}{\partial \mathrm{t}}=-\varepsilon \cdot \varepsilon_{0} \cdot \mathrm{S} \cdot \mathrm{U} \cdot \frac{\mathrm{d}_{1} \cdot \omega \cdot \cos (\omega \mathrm{t})}{\mathrm{d}_{0}+\mathrm{d}_{1} \cdot \sin (\omega \mathrm{t})^{2}}
$$

The sensors of measuring devices for measuring the CPD by the DC method are exposed to various factors that reduce the sensitivity and reliability of this method - intrinsic noises, interference from electromagnetic fields, spurious communication capacitances, etc. [9-12]. This requires careful shielding, the use of other structural design solutions in the construction of instrument sensors.

The method for measuring the CPD developed by us has the following features:

- the contact potential difference is measured by a digital oscilloscope that records the voltage and frequency of the periodically changing CPD between the controlled part and the ME of the device's sensor;

- shielding of sensor elements and wires;

- amplification of registered CPD and filtering of spurious signals;

- preparation of the surfaces of the part and the sensor for measuring the CPD.

We chose a Micsig TO1104 tablet type portable digital oscilloscope with a sensitivity of measuring electric voltage of $0.5 \mathrm{mV}$ as the recorder of CPD. The authors connected the sensor connected to the oscilloscope themselves. The main part of the design of the sensor of the measuring device of the CPD is an electronic circuit that allows the IE of the sensor in contact with the metal part to operate in self-oscillating mode. In addition, the sensor includes a preliminary signal amplifier. The CPD measurement complex developed by the authors is shown in Figure 1 .

The circuit diagram of the sensor is shown in Figure 2. The sensor housing (Figure 3) was designed in the Solid Works program and printed on a Picaso3D Designer 3D printer. The sensor housing is designed to provide a gap between $\mathrm{ME}$ and $\mathrm{CO}$ equal to $0.5 \mathrm{~mm}$ at rest of ME. The indicated design features of the sensor make it possible to perform a reliable measurement of the 
CPD of metals. The authors continue to improve the design of the sensor for measuring the CPD of metal parts of machines.

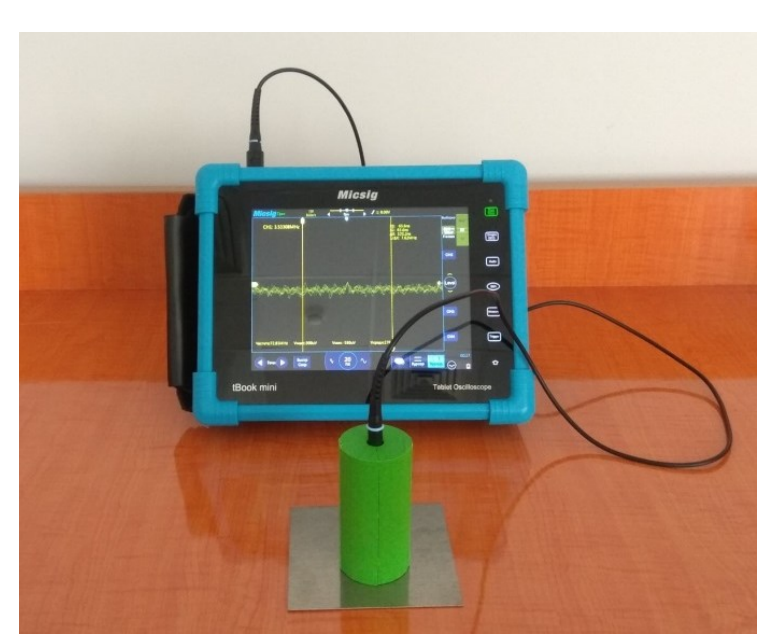

Fig.1. Complex for measuring the contact potential difference of metal parts.

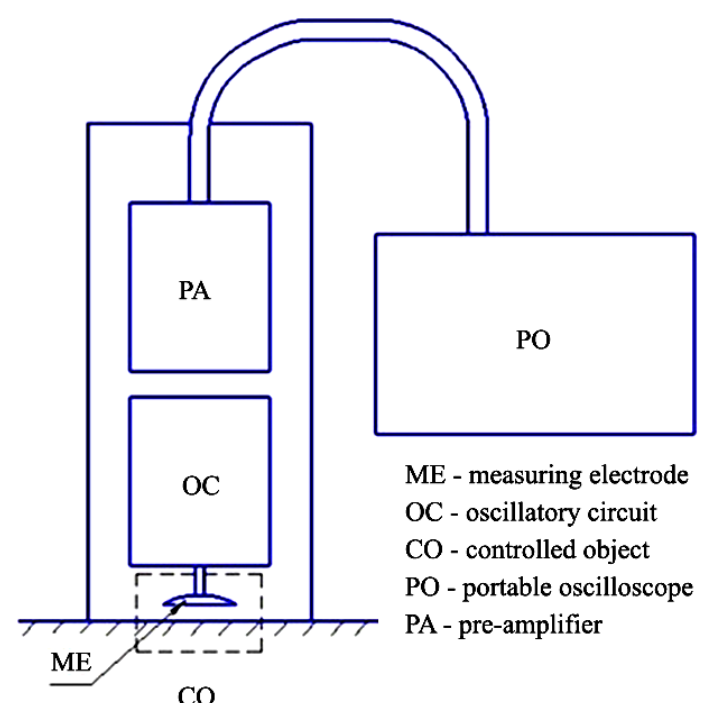

Fig.2. Schematic diagram of the sensor.

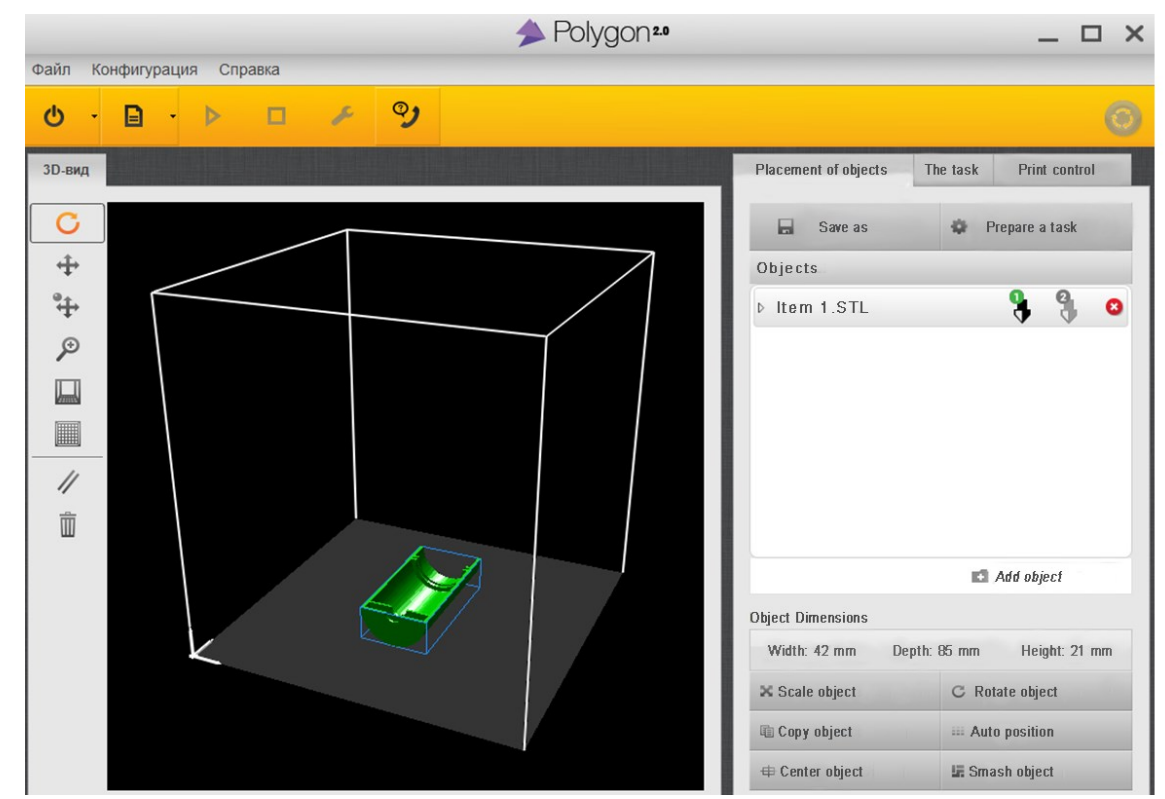

Fig.3. Polygon 2.0: Picaso3D Designer 3D Printer Operator.

\section{Conclusion}

Developed by the authors, a portable measurement complex of the CPD based on a portable digital oscilloscope allows non-destructive testing of the surface of metal parts in the process of their production, operation or repair to be carried out continuously. The results of measurements of the contact potential difference of metals were processed by methods of mathematical statistics. The results of experimental studies have shown a direct effect of changes in ambient temperature on the contact potential difference and the electrons work function of metal samples, which has an average 
correlation It is found that atmospheric pressure and relative humidity have a weak effect on the contact potential difference and the electrons work function of the metals under study, their influence can be neglected. The effect of equilibrium and non-equilibrium environmental parameters on the contact potential difference and electrons work function of metal samples is studied. The results confirming the reduction of the contact potential difference (increase in the electrons work function) of metals, as well as an increase in the mean square deviation of the measurement results under non-equilibrium environmental conditions are obtained.

Our further work will consist in the development of a technique for measuring the CPD on the surface of machine parts [13-15].

\section{Acknowledgements}

The work was performed according to the program of the Ministry of Education and Science of the Republic of Kazakhstan. Grants No. 0118PK000063 and No. Ф.0780.

\section{REFERENCES}

1 Betsofen S.Ya., Osintsev O.E., Knyazev M.I., Dolgova M.I., et al. Quantitative phase analysis of Al-Cu-Li-Mg alloys. Bulletin of the Moscow Aviation Institute. 2016, Vol. 23, No. 4, pp. 181-188.

2 Musokhranov M.V. Technological support for the quality of the surface layer of the guiding elements of mechanical engineering. Diss. of Techn. Sciences Cand. Moscow, Bauman MSTU, 2006, 138 p.

3 Zharin A.L., Gusev O.K., Svistun A.I., Tyavlovsky A.K. Friction surface control by contact potential difference methods // Izvestiya TulGU. Technical science. 2011. Issue. 5. Part 2. - P. 286-295.

4 Kocharov E.A. Physical methods in the control of materials and the development of high technology. Moscow, VVIA named after prof. N.E. Zhukovsky. 2008, 447 p.

5 Kryachko V.V., Levin M.N., Tatarintsev A.V., et al. A non-contact method for studying the charge state of a semiconductor - insulator interface. Journal of Technical Physics. 2004, Vol. 74, Issue 10, pp. 128 -133 .

6 Loskutov S.V. Regularities of the formation of the energy relief of a metal surface. News of the Zaporizhzhyan State University. 1999, No. 1, pp. 138 - 142.

7 Petrov L.M., Plikhunov V.V. Determination of the energy state of the surface of structural metallic materials after technological influences. Aviation industry. 2012, No. 1, pp. $22-26$.

8 Grachev A.V., Churakov P.P. Converter of parameters of non-contact capacitive sensors for conductodielcometric measurements // Bulletin of the Samara Scientific Center of the Russian Academy of Sciences. 2016. Vol. 18. No. 4 (7). - P. 1363-1368.

9 Tyavlovsky A.K., Zharin A.L. Analysis of the method of measuring the surface potential of dielectrics according to the scheme of current compensation. Instruments and methods of measurements. 2011, No. 2 (3), pp. $136-144$.

10 Kompaneyets I.V., Komolov V.M., Shkilko A.M. Assessment of the sensitivity of the contact potential difference meter. Bulletin of NTU "KhPI": Series "New Solutions in Modern Technologies". 2010. No. 46 , pp. $89-94$.

11 Kolesnikov V.P. Electrometry. Theoretical foundations of electrometry methods. - Perm, 2019. - 264 p.

12 Panteleev K.V. Methods and means of measuring the contact potential difference based on the analysis of the compensation dependence of the Kelvin probe. Abstract. Diss. of Candidate of Technical Sciences. Minsk: Belarusian Nat. tech. Univ. 2016, 26 p.

13 Oleshko V.S., Yurov V.M. Fluctuation of the contact potential difference in determining the work function of electrons from structural materials by the ionization method. Proceeding of the $10^{\text {th }}$ Intern. Conf. "Chaos and structures in nonlinear systems. Theory and experiment", Almaty, 2017, pp. $165-169$.

14 Oleshko V.S., Guchenko S.A., Yurov V.M. Determination of the surface energy of solid metals. Proceeding of the XIV Int. Scientific Conf. "Solid State Physics, Functional Materials, and New Technologies" (FTT-2018), Bishkek-Karaganda, 2018, pp. 124-128.

15 Yurov V.M., Oleshko V.S. The impact of the environment on the contact potential difference of metal machine parts. Eurasian Physical Technical Journal. 2019, Vol. 16, No.1 (31), pp. 99 - 108. 